\title{
Recorrido geológico y mineralógico por la comarca de Gúdar-Javalambre: desde Mosqueruela a Linares de Mora a Valdelinares y a Rubielos de Mora
}

Josep Maria Mata-Perelló

Andrés Pocoví Juan

Joaquim Sanz Balagué

Jaume Vilaltella

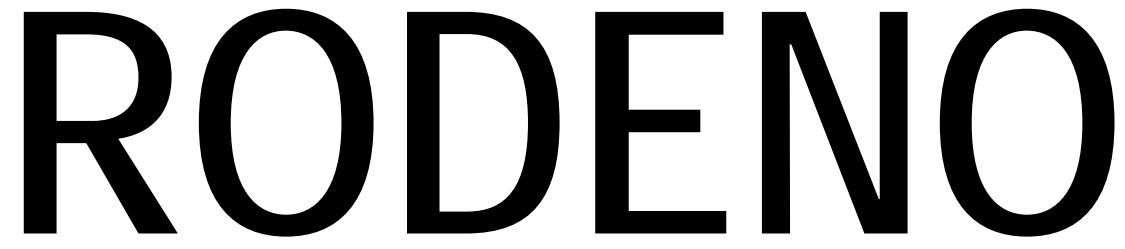

\section{REVISTA DE GEOLOGÍA ARAGONESA}

\section{n. 1}

ENERO 2015 


\title{
RECORRIDO GEOLÓGICO Y MINERALÓGICO POR LA COMARCA DE GÚDAR-JAVALAMBRE: DESDE MOSQUERUELA A LINARES DE MORA A VALDELINARES Y A RUBIELOS DE MORA
}

\author{
Josep Maria Mata-Perelló \\ Museu de geologia Valentí Masachs, Escola Politècnica Superior d'Enginyeria de Manresa \\ (EPSEM), Universitat Politècnica de Catalunya · BarcelonaTech (UPC), 08272 Manresa, Spain
}

\author{
Andrés Pocovi Juan \\ Universidad de Zaragoza, Departamento de Ciencias de la Tierra ( DCT- UZ) \\ Joaquim Sanz Balagué \\ Departament d'Enginyeria Minera i Recursos Naturals (EMRN), Escola Politècnica Superior \\ d'Enginyeria de Manresa (EPSEM), Universitat Politècnica de Catalunya - BarcelonaTech \\ (UPC), 08272 Manresa, Spain
}

\section{Jaume Vilaltella Farràs.}

Sociedad Española para la Defensa del Patrimonio Geológico y Minero (SEDPGYM)

Palabras clave: patrimonio minero; patrimonio geológico; Zaragoza

\section{Resumen}

En este caso, el presente itinerario se desarrollará totalmente en la denominada Rama Aragonesa de la Cordillera Ibérica (que ya no se abandonará). En este tramo se irán encontrando afloramientos de los materiales mesozoicos (del Triásico, del Jurásico y del Cretácico). Más concretamente, se realizará en por la denominada Cuenca del Maestrazgo, siempre, sin abandonar la denominada Zona de Enlace de la Cordillera Ibérica con el Sistema Mediterráneo; aunque en todos los casos van a predominar las estructuras ibéricas (WNWESE) sobre las "catalanas" (NNE-SSW), al hallarnos plenamente dentro del dominio ibérico.

Por otra parte, este itinerario discurrirá por una sola de las comarcas de Teruel, concretamente por la de Gúdar - Javalambre, aunque esta comarca incluye sectores tradicionalmente del Maestrazgo, como los del término de Mosqueruela. 


\section{Objetivos generales de este itinerario}

Los objetivos fundamentales que se pretenden conseguir con la realización de este itinerario, se pueden concretar en los siguientes aspectos generales:

1. Observación de los materiales mesozoicos (del Triásico, Jurásico y Cretácico) que encontraremos por diferentes lugares del recorrido. Estos materiales forman parte del Sistema Ibérico en la Sierra de Gúdar.

2. Observación de la estructura, en este recorrido, de la denominada Cuenca del Maestrazgo; y también del conjunto del Sistema Ibérico en esta Zona de Enlace de la Cordillera Ibérica con el Sistema Mediterráneo.

3. Observación de las diferentes mineralizaciones que iremos encontrando a lo largo del recorrido, en especial de las siguientes:

3A) de las mineralizaciones estratiformes de $\mathrm{Pb}-\mathrm{Zn}$, que encontraremos por distintos lugares de los municipios de Linares de Mora y de Valdelinares.

3B) de las mineralizaciones ferruginosas asociadas a hard-grounds, que encontraremos en la Sierra de Férríz, en el término de Linares.

4. Observación de las explotaciones mineras desarrolladas sobre las mineralizaciones anteriores, y también sobre otras, a lo largo del recorrido del itinerario.

5. Observación de los impactos producidos sobre el Medio Natural, como consecuencia de las explotaciones anteriores.

6. Observación (si se da el caso) de las restauraciones llevadas a cabo, con la finalidad de mitigar los impactos anteriores.

7. Observación, a lo largo de todo el recorrido, de los distintos PIG (Puntos de Interés Geológico) y de los PIM (Puntos de Interés minero), integrantes de nuestro Patrimonio Geológico y Minero.

\section{Antecedentes bibliográficos}

Solamente tenemos conocimiento de un itinerario geológico y minero que discurre por estos sectores; aunque de una forma un tanto marginal. Se trata de MATA-PERELLÓ (2003). Recientemente y por lo que corresponde al tramo final del recorrido, haremos mención del trabajo de MATA - PERELLÓ (2014).

Sin embargo, tenemos antecedentes nuestros, en relación a las mineralizaciones presentes en esta zona, tanto a las situadas en esta comarca como en las vecinas (a las del Maestrazgo y Cuencas Mineras). Se trata de MATA-PERELLÓ (1991a, 1991b y 1991c)

En relación con las características geológicas citaremos el trabajo de PEÑA et altri (1984). Asimismo, en relación al patrimonio geológico citaremos el trabajo de BURILLO (1996), así como el publicado por la GADMA (2001).

Todos estos trabajos se hallaran debidamente relacionados en el apartado de REFERENCIAS BIBLIOGRÁFICAS, que figura al final del presente trabajo. 


\section{Recorrido del itinerario}

El recorrido de este itinerario, se iniciará en las cercanías de la población de Mosqueruela, con una hijuela hacía una de las numerosas explotaciones de "losas" y "losetas". Luego, desde esa población, el recorrido se encaminará hacía el Alto de Linares. En este tramo se efectuaran diversas paradas.

Tras ello, el recorrido llegará hasta las inmediaciones de Linares de Mora; aunque se realizará una nueva hijuela hacía el término de Valdelinares. A lo largo de esta hijuela se harán dos paradas para observar las antiguas explotaciones mineras de Linares y Valdelinares, en el Barranco de la Gitana.

A continuación, tras volver a atrás, se efectuará una parada en las cercanías del Pino del Escobón, para observar las minas de $\mathrm{Pb}-\mathrm{Zn}$, situadas en sus inmediaciones; así como los restos del patrimonio minero.

Luego, el recorrido se encaminará hacia Linares de Mora, efectuándose algunas paradas por los alrededores del pueblo. Tras ello, el recorrido continuará hacia el SW, con la intención de llegar hasta el collado del Pino de San Bartolomé, en donde finalizará tras hacer una hijuela hasta las minas de hierro de la Sierra de Férriz.

Finalmente, se encaminará hacía Rubielos de Mora, por donde se efectuará la última parada.

\section{Advertencias previas}

Al igual que en otras ocasiones, en los recorridos de RECERCA GEOLÒGICA I NATURALİSTICA..., si se dispone del tiempo suficiente, podrán realizarse todas las paradas e hijuelas propuestas. En caso contrario, recomendamos prescindir de las calificadas con el nombre de PARADAS - CONDICIONALES.

Así se presentará un recorrido aproximado, por diferentes lugares de la comarca de Gúdar Javalambre (especialmente por los sectores más orientales de la misma), con una serie de PARADAS situadas entre las poblaciones de Mosqueruela y Linares de Mora, con una pequeña incursión por el término municipal de Valdelinares.

Aun así, el recorrido podría variarse en función del estado de conservación de los caminos, de la situación meteorológica, o por hallar sobre la marcha lugares más interesantes, para poder efectuar las pertinentes observaciones.

Posiblemente, en algunos tramos del recorrido se pasará por caminos en mal estado de conservación, circunstancia por la cual será mejor hacerlos a pie. Este es el caso del camino de aproximación a alguna de las minas de Linares o de Valdelinares.

En cualquier caso, y como es natural, es necesario tener un absoluto cuidado y respeto hacia el Medio Natural, a lo largo de todo el recorrido. 


\section{Descripción del itinerario}

Como de costumbre, estructuraremos el recorrido del itinerario en una serie de PARADAS, que a continuación iremos viendo. En cada una de estas paradas haremos un breve comentario (geológico o mineralógico, según acontezca). Por otra parte, en cada caso indicaremos (entre paréntesis) la hoja topográfica en donde se halla la parada. Para ello, utilizaremos las hojas del Instituto Geográfico y Catastral de España, publicadas a la escala de 1:50.000. En este caso, las hojas serán las siguientes: 568 (o de Alcalá de la Selva), 569 (o de Vilafranca del Cid Mosqueruela), 590 (o de la Puebla de Valverde), 591 (conocida también con el nombre de Mora de Rubielos) y 592 (o de Villahermosa del Río). Así pues, la relación de las paradas que componen el recorrido de este itinerario, son las siguientes:

PARADA 1 - CONDICIONAL. EXPLOTACIONES DE LOSAS DEL CORRAL DE LA BALSA, EL BOIRO, (término municipal de Mosqueruela, comarca de Gúdar-Javalambre). (Hoja 569).

El recorrido de este itinerario habrá empezado en la población turolense de Mosqueruela, desde donde será necesario salir hacía el levante, por la carretera autonómica que conduce hacía la cercana población de la Iglesuela del Cid. Al llegar a las inmediaciones del antiguo Km 64 (a unos $2 \mathrm{Km}$ del pueblo), es necesario tomar un camino que conduce a unas explotaciones de losas. Ahí haremos la primera parada de este recorrido.

Este recorrido se habrá efectuado por entre afloramientos de los materiales mesozoicos del Cretácico. Estos materiales son los que afloran en el lugar de la parada. En este caso se trata de calizas arenosas.

Precisamente, estos materiales están siendo explotados (tanto en este lugar, como en muchos otros del municipio de Mosqueruela). En todos los lugares, se destinan a la obtención de losetas para la construcción. (fotografia 1).

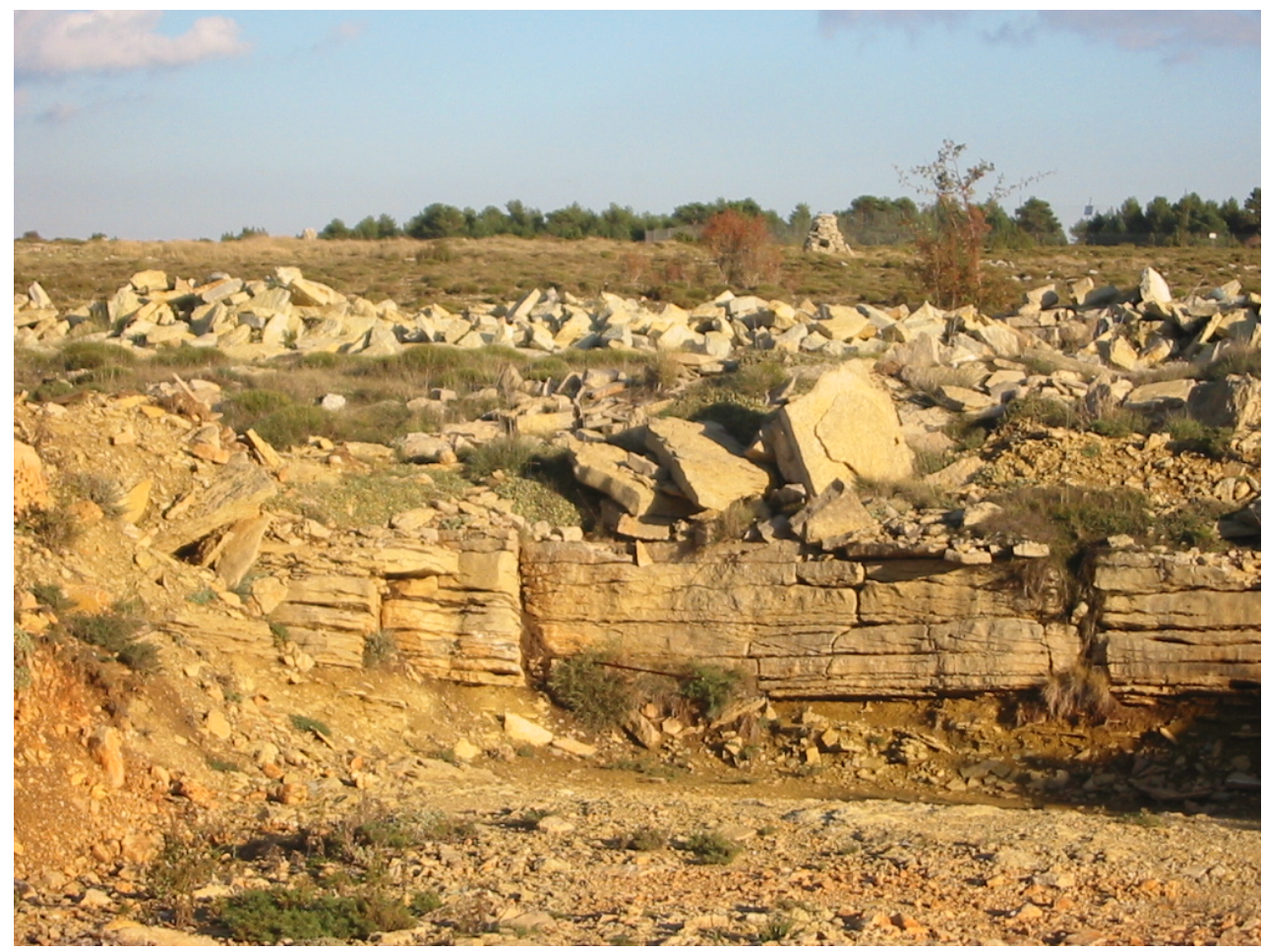

Fotografía 1. Vista general de una de las explotaciones de losa del municipio de Mosqueruela 
PARADA 2. POLJÉ DE MOSQUERUELA, CRUCE DE LA CARRETERA A PUERTOMINGALVO CON LA DE LINARES DE MORA, (término municipal de Mosqueruela, comarca de Gúdar-Javalambre). (Hojas 569 y 592).

Desde la parada anterior es necesario retornar hacia la cercana población turolense de Mosqueruela, desde donde será necesario salir hacia el poniente, por la carretera autonómica que conduce hacía la cercana población de Linares de Mora.

En este recorrido, justo a la salida del pueblo de Mosqueruela, empieza a observarse a la izquierda de la carretera una extensa planicie de varios kilómetros de longitud; desde el pueblo hasta casi $1 \mathrm{Km}$ más allá del cruce de la carretera local que conduce a Puertomingalvo. En este mismo lugar podemos realizar la primera parada de este recorrido.

Esta extensa planicie corresponde al poljé de Mosqueruela, uno de los mejores ejemplos de estas formaciones kársticas. En este caso, se halla situado entre afloramientos de las calizas mesozoicas del Cretácico. Este lugar forma parte del catálogo de PIG (Puntos de Interés Geológico) de Aragón.

Este extenso poljé de Mosqueruela es similar a los vecinos de Vilafranca del Cid y Vistabella del Maestrat, situados en la vecina provincia de Castelló, en las comarcas del Baix Maestrat y del Alcalatén, respectivamente. En conjunto, los tres, representan unos de los poljés más interesantes de toda la Península Ibérica. (fotografia 2).

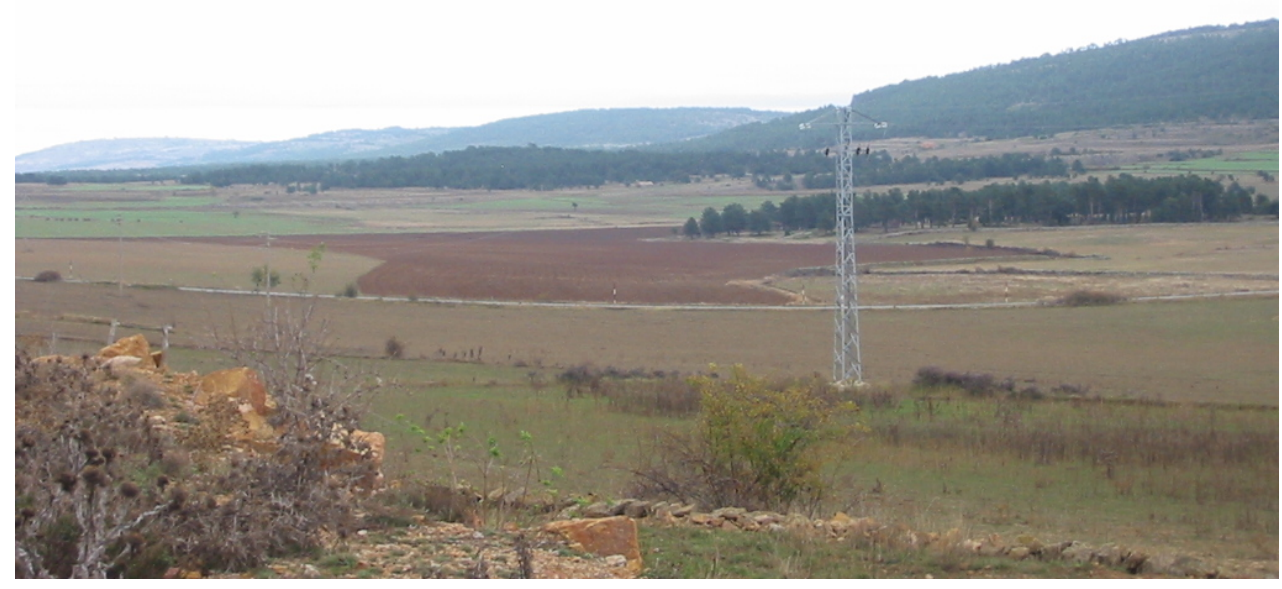

Fotografía 2. El poljé de Mosqueruela, desde su sector más occidental 
PARADA 3. CABECERA DEL BARRANCO DEL REBOLLAR, (término municipal de Linares de Mora, comarca de Gúdar-Javalambre). (Hoja 591).

Desde la parada anterior, es necesario continuar hacía Linares de Mora. A algo más de $1 \mathrm{Km}$ del cruce de Puertomingalvo, puede efectuarse una nueva parada, a la izquierda de la carretera.

En este recorrido habremos ido encontrando los materiales cretácicos mencionados anteriormente. Estos materiales son también los que aparecen en el lugar de la presente parada.

En este lugar, mirando hacia el SE se hace ostensible un profundo barranco, que surge de la planicie que hemos estado recorriendo hasta ahora. Se trata de un barranco surgido por el colapso de una parte de esta planicie kárstica, similar a la ubicada en el Barranc de la Fos (de Vilafranca del Cid), por ejemplo. En este caso, este Barranco del Rebollar es tributario del río Linares, que más abajo pasa a denominarse río de Villahermosa. FOTOGRAFÍA 3

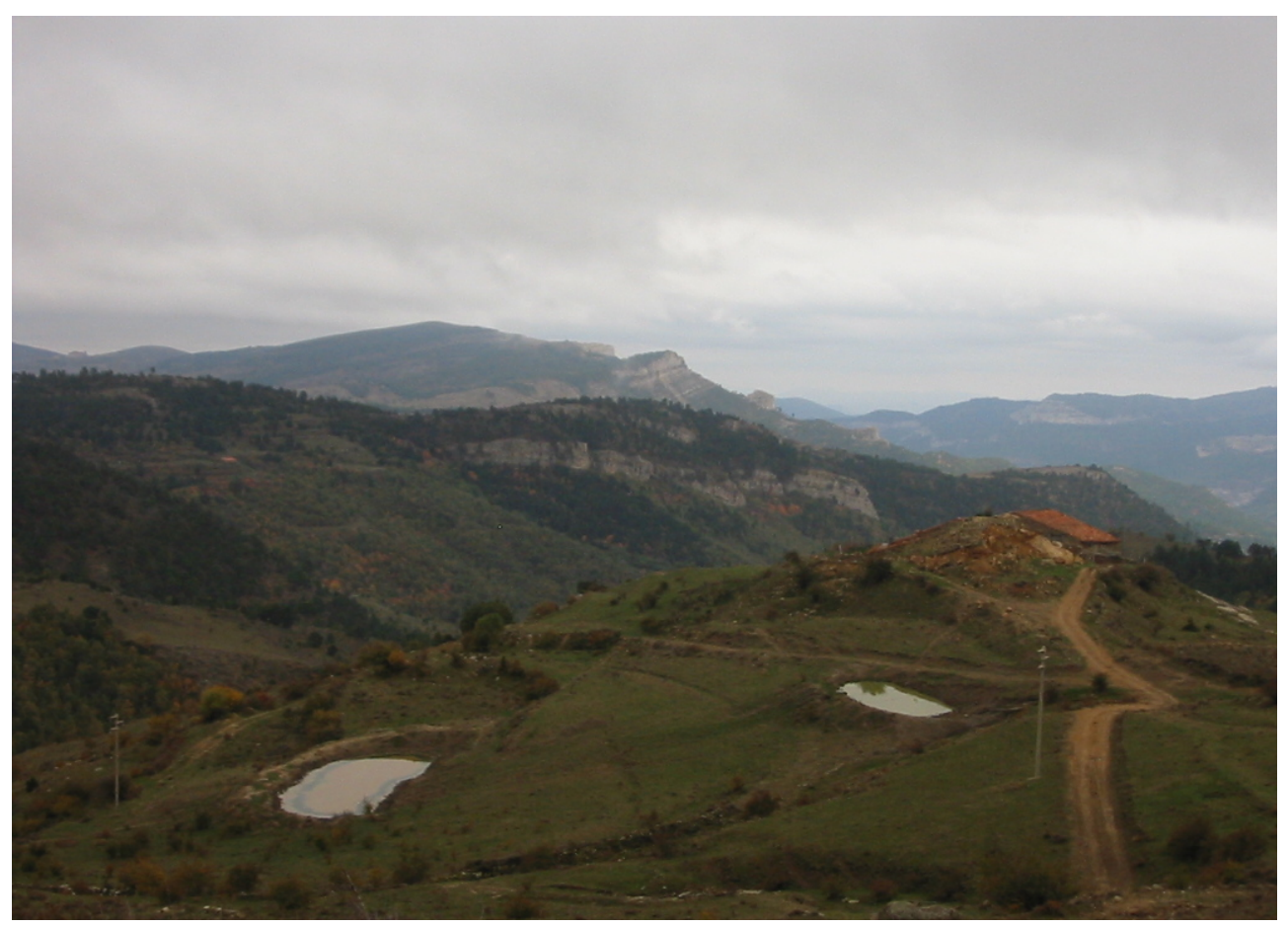

Fotografía 3. El Barranco del Rebollar (cuenca del río Linares) al poniente del Poljé de Mosqueruela 
PARADA 4 - CONDICIONAL. MIRADOR DEL PUERTO DE LINARES, (término municipal de Linares de Mora, comarca de Gúdar-Javalambre). (Hoja 591).

Tras efectuar la parada anterior, cabe la posibilidad de hacer un pequeño recorrido, yendo hacia el poniente. Así, pronto se llegará y superará el Puerto de Linares. En su caída occidental, puede realizarse una nueva parada en las inmediaciones del Mirador.

En este recorrido habremos ido encontrando afloramientos de los materiales mesozoicos del Cretácico, ya citados en las paradas anteriores. Estos son también los materiales que aparecen en este sector.

Desde aquí, puede observarse el impresionante y profundo valle del río Linares (que al cambiar de provincia, cerca de aquí) pasa a llamarse río de Villahermosa. Este río, tributario del Mijares, sigue claramente una dirección ibérica.

PARADA 5. MINA RESTAURACIÓN, (término municipal de Linares de Mora, comarca de Gúdar-Javalambre). (Hoja 568).

Tras realizar la parada anterior, es necesario continuar por la carretera hacía el pueblo de Linares de Mora. Casi al llegar, a menos de $1 \mathrm{Km}$, encontraremos la carretera que conduce a Valdelinares. A unos $5 \mathrm{Km}$ del cruce, y a unos $7 \mathrm{Km}$ de la parada anterior, en la orilla opuesta del Río Linares, (por el que vamos ascendiendo, tras haberlo cruzado), pueden observarse varias minas. Nosotros haremos la parada en la Mina Resurrección. Sin embargo, antes de llegar a estas minas, habremos ido superando otras, que se hallan en la parte opuesta de la carretera; así, pueden observarse hasta un total de 19 bocaminas, agrupadas en tres concesiones importantes: Avecilla, Antonia y Resurrección, de la parte inferior a la superior del barranco. Nosotros haremos la parada en esta última, la más importante y la más accesible desde la carretera.

Las características de este tramo recorrido ahora desde la parada anterior, son del todo similares a los efectuados anteriormente en las otras paradas. En efecto, hemos estado atravesando niveles carbonatados calcáreos, pertenecientes al Aptiense de la Sierra de Gúdar.

Por otra parte, en este lugar entre las calizas y dolomías aptienses, se halla una mineralización estratiforme de $\mathrm{Pb}-\mathrm{Zn}(\mathrm{Fe})$. Así, afloran unas calizas arrecifales aptienses. En este lugar los niveles calcáreos se hallan muy dolomitizados. Asociada a ellas hay una mineralización estratiforme de $\mathrm{Pb}-\mathrm{Zn}$ (Fe), que ha sido explotada para el beneficio de los minerales de los minerales de esos metales.

Así, los minerales de Pb-Zn presentes son los siguientes: ESFALERITA, GALENA, CERUSITA, HIDROCINCITA, SMITHSONITA y ANGLESITA. Entre ellos predominan los de plomo, especialmente la galena.

Junto a los anteriores, también se encuentran los minerales de hierro siguientes: GOETHITA, HEMATITES, SIDERITA y SIDEROGEL, más minoritarios. Asimismo, también hay: CALCOPIRITA (indicios), CALCITA, DOLOMITA y BARITINA.

Aquí, en este caso se puede entrar ligeramente en la mina, pero cabe extremar al máximo las precauciones, dada la inestabilidad de la misma, ante más que posibles desprendimientos. (fotografies 4 y 5 ). 


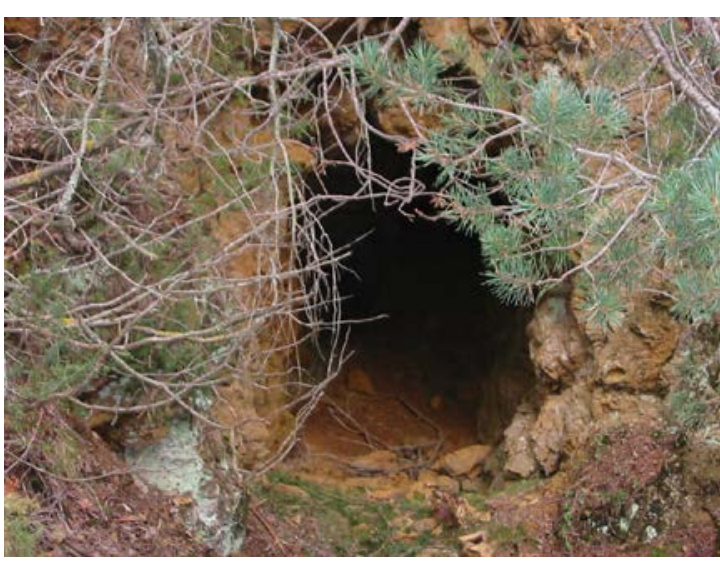

Fotografía 4. Una de las bocaminas superiores de la Mina Restauración

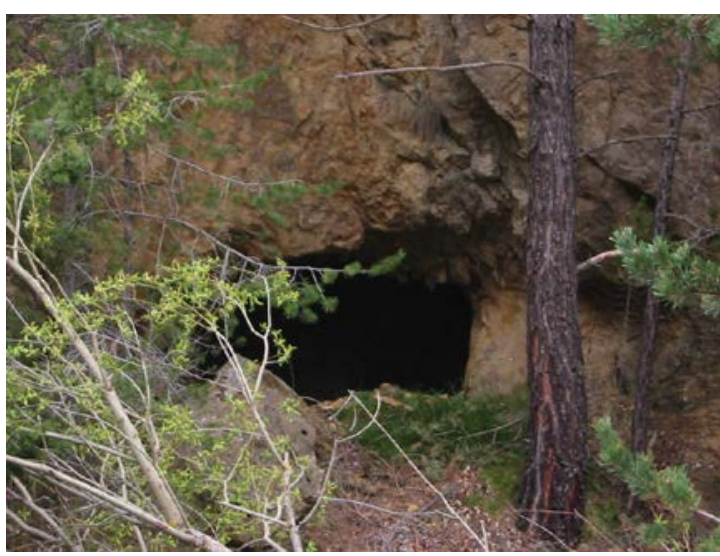

Fotografía 5. Una de las bocaminas inferiores de la Mina Restauración

Por otra parte, cabe indicar que existe un importante patrimonio minero relacionado con estas minas. Lamentablemente, debido a los desprendimientos antes mencionados, se encuentra en muy mal estado de conservación. (fotografías 6 y 7).

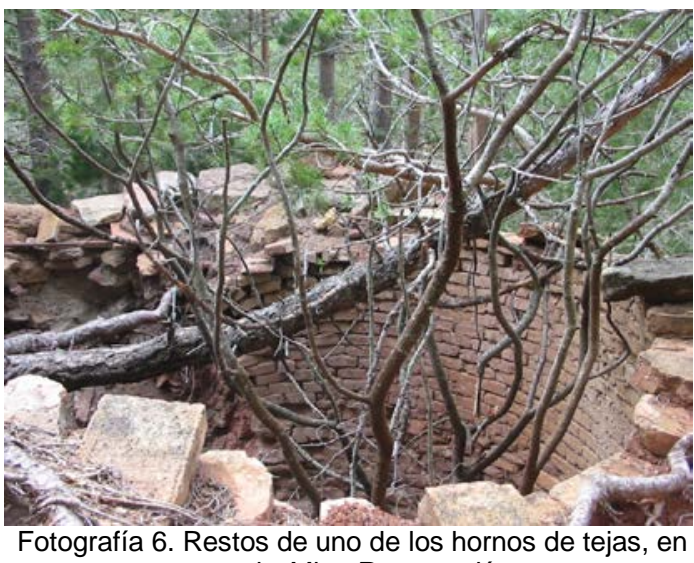
la Mina Resurección

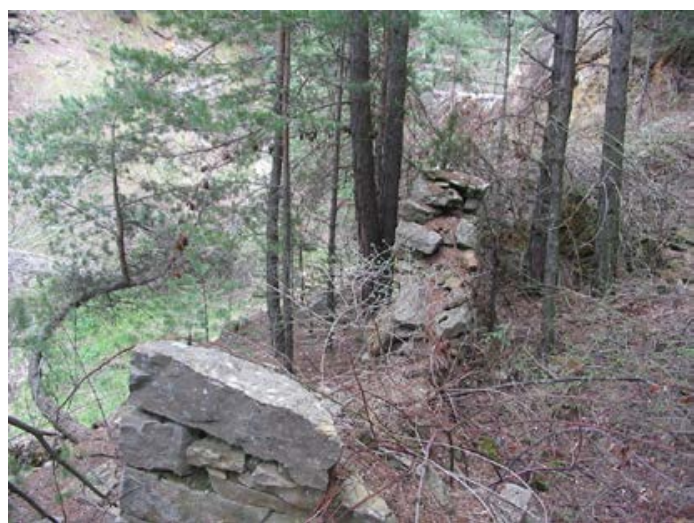

Fotografía 7. Restos de uno de los edificios, quizás de viviendas de la Mina Resurección 
PARADA 6 - CONDICIONAL. MINA DEL BARRANCO DE LA GITANA, (término municipal de Valdelinares, comarca de Gúdar-Javalambre). (Hoja 568).

Tras hacer la parada anterior, cabe seguir por la carretera hacía el pueblo de Valdelinares. Al llegar a las inmediaciones del Mas de Guarque y del Mas de Gimeno (uno a cada lado de la carretera), conviene dejarla, para remontar casi $1 \mathrm{Km}$ el Barranco de la Gitana, hasta llegar a la mina. Ahí podemos hacer una nueva parada, a unos $1,5 \mathrm{Km}$ de la anterior.

Las características del recorrido de este tramo son totalmente similares a las de la parada anterior. Efectivamente, habremos ido encontrando afloramientos de los materiales carbonatados del Aptiense, dentro de la Sierra de Gúdar.

Por otra parte, por lo que concierne al lugar de la parada, cabe decir que afloran como en los casos anteriores, las calizas dolomitizadas del Aptiense. Asimismo, en relación con ellas se halla una mineralización estratiforme de $\mathrm{Pb}-\mathrm{Zn}(\mathrm{Fe})$, con presencia mayoritaria de GALENA y de SMITHSONITA, junto a los otros minerales mencionados en las paradas anteriores.

PARADA 7 - CONDICIONAL. LAS "PALSAS" DEL BARRANCO DE ZOTICOS, (término municipal de Valdelinares, comarca de Gúdar-Javalambre). (Hoja 568).

Luego, tras efectuar la parada anterior, es necesario llegar finalmente a la población de Valdelinares (una de las más altas de la Península). Aquí, en el mismo pueblo, nos convendrá ir hacía el Nacimiento del Barranco de Zoticos, o también al Barranco de las Ranas. En cualquier caso, habremos efectuado un recorrido no superior a los $4 \mathrm{Km}$, para llegar hasta aquí.

En este recorrido, habremos circulado a través de los afloramientos de los materiales carbonatados mesozoicos que configuran la Sierra de Gúdar por estos contornos. Estos materiales son en su mayor parte de edad cretácica, perteneciendo al Aptiense en la mayoría de los casos

En esta parada puede realizarse una interesante observación del modelado periglaciar. Así, por una parte, en el Nacimiento del Barranco de Zoticos, pueden verse las denominadas "palsas". Son montículos más o menos circulares de tierra de dimensiones decimétricas a métricas, que se forman al congelarse las aguas que empapan las tierras.

Por otra parte, en el Barranco de las Ranas, pueden observarse unos deslizamientos de las laderas, provocadas por las aguas de imbibición, al congelarse. Ello ha dado lugar a la formación de unos fértiles bancos de solifluxión. 
PARADA 8. EL PINO DEL ESCOBÓN, (término municipal de Linares de Mora, comarca de Gúdar-Javalambre). (Hoja 591).

Tras efectuar la parada anterior, es necesario retornar a la carretera, para poder llegar así de nuevo a las cercanías de Linares de Mora. Al llegar al inicio del camino que conduce al Pino del Escobón, a la derecha de la carretera (subiendo hacía Linares) será necesario tomarlo. Al llagar ahí haremos una nueva parada., a unos $10 \mathrm{Km}$ de la anterior, y a unos 4’ 5 de la Mina Restauración (PARADA 5).

En este recorrido, hemos vuelto a cortar los afloramientos de los materiales mesozoicos ya citados en los recorridos anteriores.

En este lugar, entre los afloramientos de los materiales carbonatados cretácicos, aparece un pino monumental, muy conocido en la zona y en todo el maestrazgo. Se trata del Pino del Escobón, que bien vale una parada para observarlo. (fotografia 8).

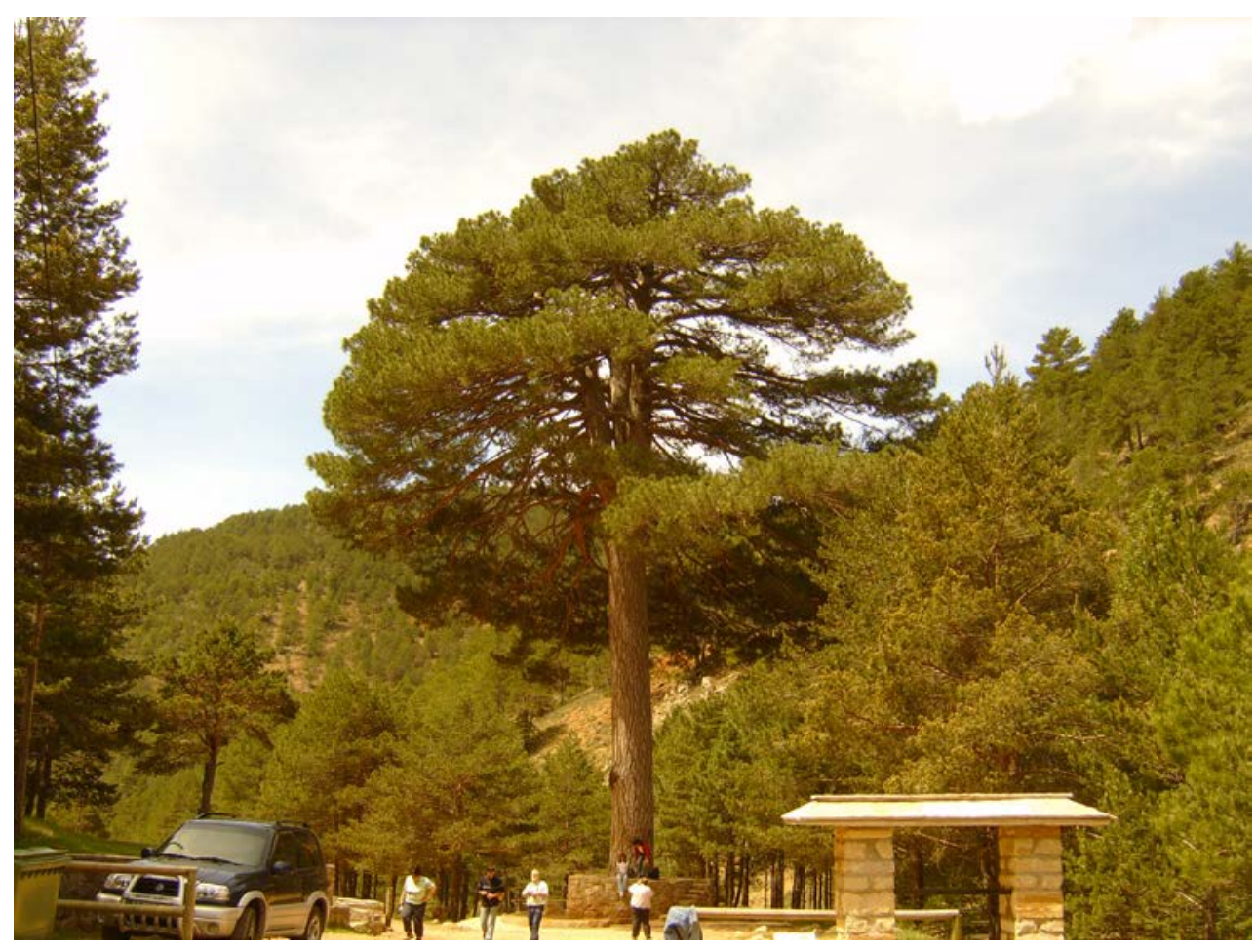

Fotografía 8. Pino del Escobón 
PARADA 9. MINA RESURRECCIÓN, (término municipal de Linares de Mora, comarca de Gúdar-Javalambre). (Hoja 591).

Tras efectuar la parada anterior, es necesario recorrer a pie una senda ascendente, por el lado septentrional del barranco. Por esta senda, en unos 10 minutos, llegaremos a las importantes bocaminas de la Mina Resurrección, en donde efectuaremos una nueva parada, dentro de esta zona minera.

En este recorrido, hemos ido encontrando los materiales carbonatados calcáreos, ya mencionados en la parada anterior. Estos niveles mesozoicos del Cretácico, forman parte de la Sierra de Gúdar.

En este lugar afloran de nuevo las calizas arrecifales aptienses, que ya hemos mencionado en la parada anterior. En este lugar los niveles calcáreos se hallan muy dolomitizados. Asociada a ellas hay una mineralización estratiforme de $\mathrm{Pb}-\mathrm{Zn}$ ( $\mathrm{Fe})$, que ha sido explotada para el beneficio de los minerales de los minerales de esos metales. Esta mineralización es similar a la que hemos visto en la PARADA 5, en la Mina Restauración. El conjunto de estas explotaciones mineras, puede verse en las FOTOGRAFIAS 9, 10, 11 y 12.

Así, los minerales de Pb-Zn presentes son los siguientes: ESFALERITA, GALENA, CERUSITA, HIDROCINCITA, SMITHSONITA y ANGLESITA. Entre ellos predominan los de plomo, especialmente la galena. Junto a los anteriores, también se encuentran los minerales de hierro siguientes: GOETHITA, HEMATITES, SIDERITA y SIDEROGEL, más minoritarios. Asimismo, también hay: CALCOPIRITA (indicios), CALCITA, DOLOMITA y BARITINA.

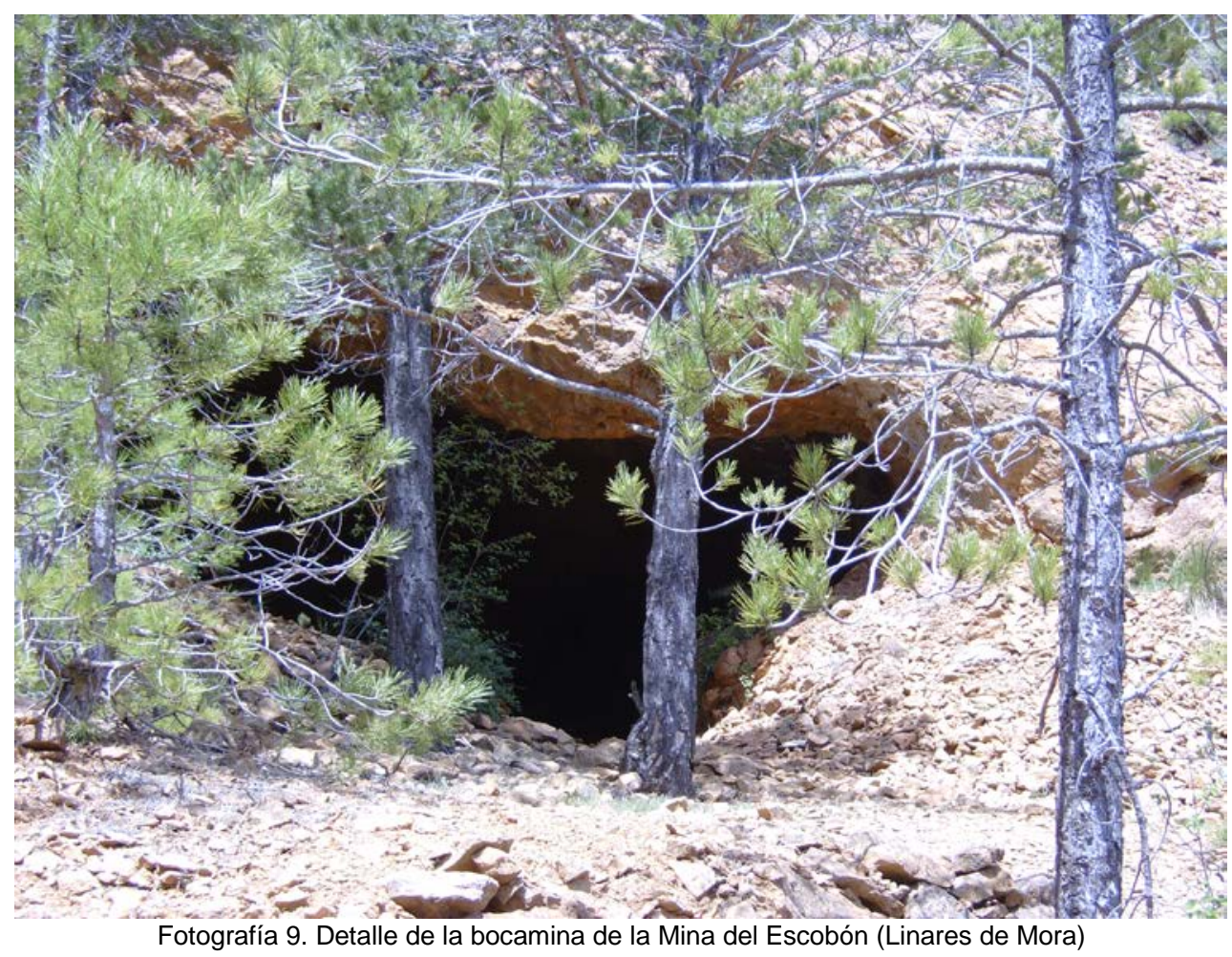




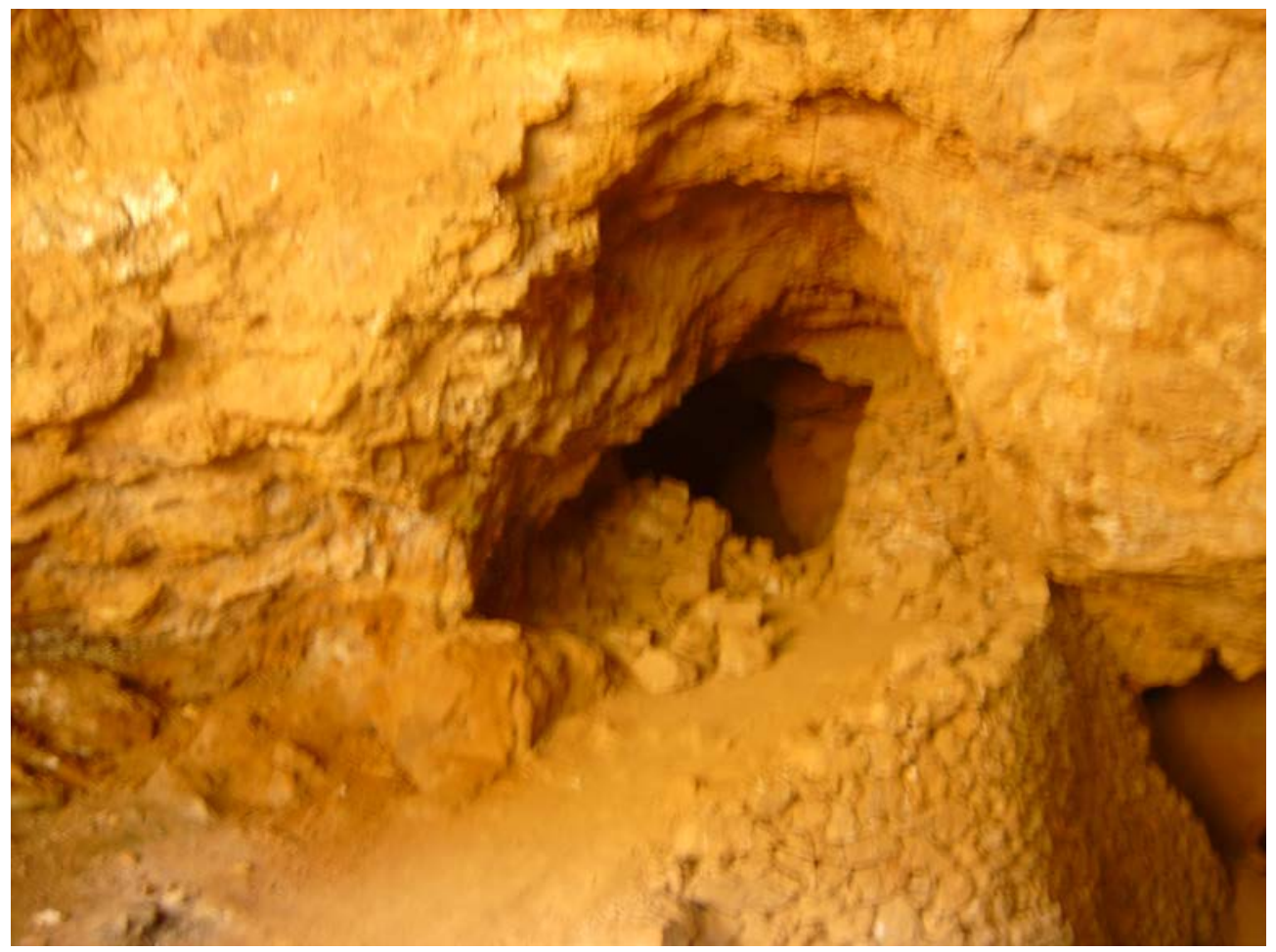

Fotografía 10. Interior de la Mina del Escobón

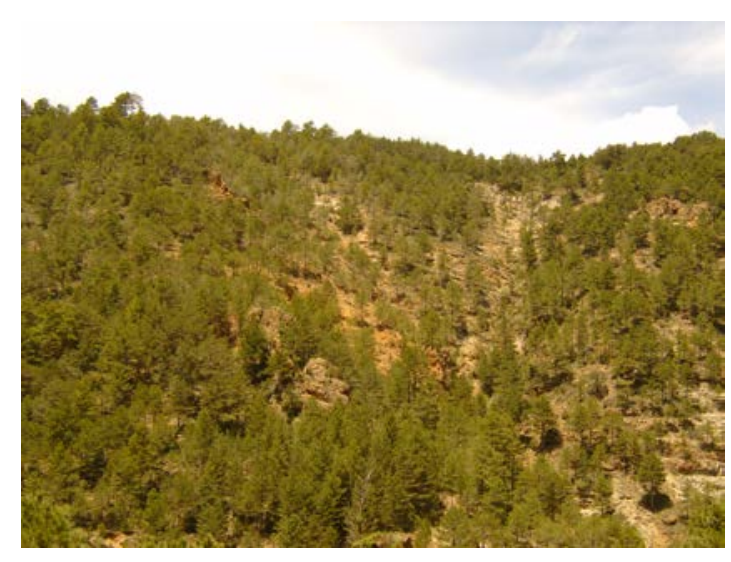

Fotografía 11. Contacto entre los niveles calcáreos y los dolomíticos

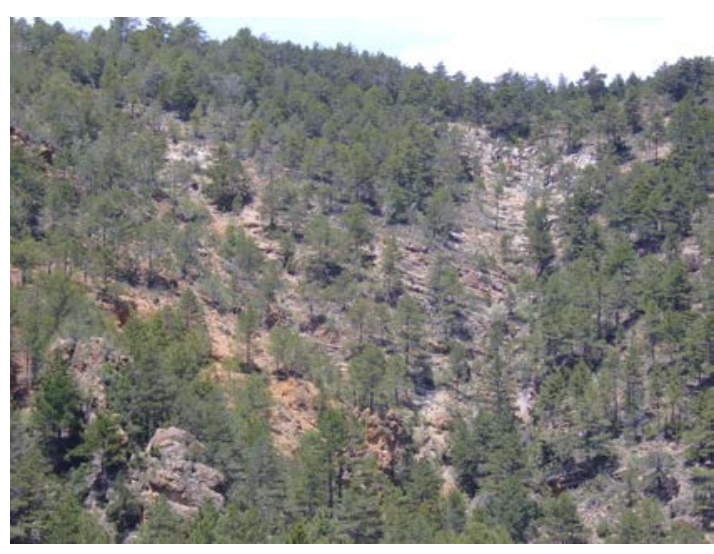

Fotografía 12. Detalle del contacto de la fotografía anterior 
PARADA 10 - CONDICIONAL. ANTIGUA HERRERÍA, (término de Linares de Mora, comarca de Gúdar-Javalambre). (Hoja 591).

Tras efectuar la parada anterior, es necesario retornar a Linares de Mora, para ir luego hacía el SW, por la carretera que conduce a Nogueruelas y a Rubielos de Mora. Al sobrepasar el puente sobre el río Linares, cabe tomar un camino por la derecha, que desciende hacía el río, pasando por debajo del puente. Luego, cabe seguir descendiendo por el río, hasta llegar a la casa del Molino de la Herrería. A partir de aquí, por bellísimos lugares, cabe ir descendiendo por el río, unos 500 metros, hasta llegar a los restos de la Ferrería. Aquí, a unos $3 \mathrm{Km}$ del pueblo, a unos dos del puente y a unos $6 \mathrm{Km}$ de la parada anterior, si acontece, podemos hacer otra.

En este recorrido habremos ido encontrando afloramientos de los materiales mesozoicos citados en las paradas anteriores, pertenecientes al Cretácico. Poco antes de llegar al puente habremos cortado un "hard-ground ferruginoso", con muchos óxidos de hierro (HEMATITES), fundamentalmente).

En este lugar, quedan los restos de una importante herrería, al estilo "catalán" que aprovechaba los minerales de hierro que se extraían de diversos "hard-grounds" cercanos. Los retos se hallan muy destrozados y creemos que sería interesante la realización de un estudio, encaminado a la preservación y excavación de los restos de esta ferrería de los siglos XVII XIX. (fotografia 13).

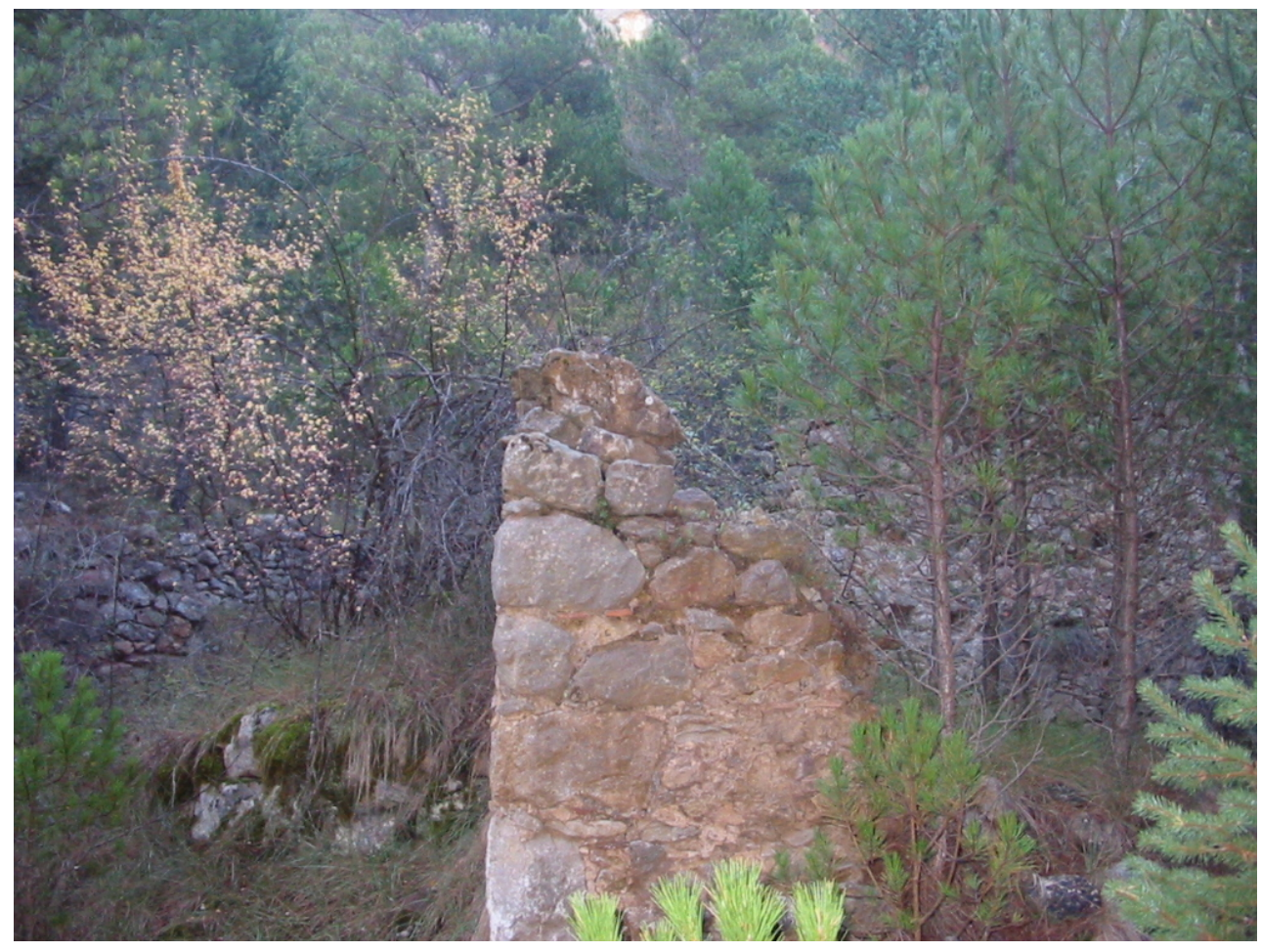

Fotografía 13. Restos de uno de los edificios de la Herrería 
PARADA 11. PINO DE SAN BERNABÉ, (término municipal de Linares de Mora, comarca de Gúdar-Javalambre). (Hoja 591).

Después de realizar la parada anterior, es necesario retornar a la carretera, para seguir hacía Nogueruelas. Tras efectuar la subida del Collado del Pino de San Bartolomé, llegaremos hasta este lugar. Aquí haremos una nueva parada, a unos $6 \mathrm{Km}$ más arriba de la anterior.

En este recorrido habremos ido encontrando afloramientos de los materiales mesozoicos, ya citados en las paradas anteriores.

En este lugar, puede efectuarse una parada para observar este árbol monumental, el Pino de San Bartolomé, bien merece la pena.

PARADA 12. MINA DE FÉRRIZ, (término municipal de Linares de Mora, comarca de GúdarJavalambre). (Hoja 591).

Después de realizar la parada anterior, es necesario tomar un camino de tierra, que se dirige hacia el Sur. Este camino conduce hacía la Casa Carbón. Desde ese camino, conviene tomar otro, que en unos $2 \mathrm{Km}$ nos conducirá hacía la Mina de Férriz. Así, desde el Pino de San Bartolomé, hasta aquí habremos recorrido unos $6 \mathrm{Km}$.

A través de este recorrido, nos hemos ido adentrando entre los afloramientos mesozoicos (fundamentalmente de los niveles carbonatados del Cretácico). Éstos materiales forman parte de la Sierra de Gúdar, perteneciente al Sistema Ibérico, por donde estamos circulando.

En este lugar afloran los niveles carbonatados calcáreos del Aptiense (Cretácico Inferior), Estos materiales son de origen arrecifal y se hallan muy dolomitizados. Asociada estos niveles hay una mineralización ferruginosa a su vez asociada a un "hard-ground". Los minerales preponderantes son los de hierro, con presencia de: GOETHITA (muy limonítica), HEMATITES (terroso), LEPIDOCROCITA (indicios, siempre limoníticos), SIDERITA y el gel SIROGEL. Junto a ellos hay indicios de otros minerales como la CALCITA, la DOLOMITA y la BARITINA.

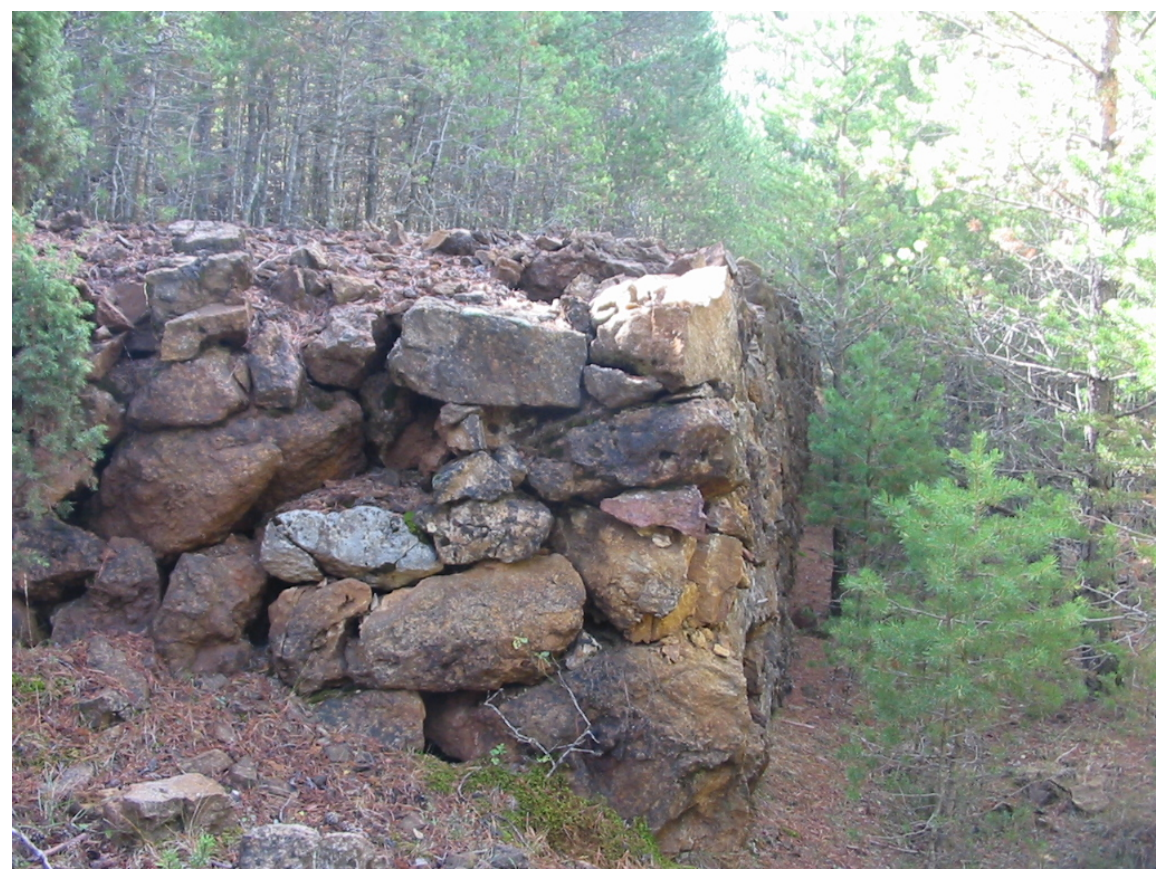

Fotografía 14. Restos del muelle de carga de las minas de hierro. 
PARADA 13. MINAS DE MARGAS BITUMINOSAS DE RUBIELOS DE MORA, MINA SAN JOSÉ, CARRETERA A - 1515, (término municipal de Rubielos de Mora, comarca de GúdarJavalambre). (Hoja 591).

Tras realizar la parada anterior, conviene retornar a la carretera que enlaza Linares de Mora con Rubielos de Mora. En llegar a ella, nos convendrá continuar hacía el último pueblo mencionado, pasando antes por Nogueruelas. Tras llegar a él, a Rubielos de Mora, convendrá superarlo, con la finalidad de llegar a las instalaciones de las antiguas explotaciones de margas bituminosas, situadas a la izquierda de la carretera A - 1515, a unos 350 metros ella y a unos $5 \mathrm{Km}$ del pueblo. Así, para llegar hasta aquí, desde la parada anterior, habremos efectuado un recorrido cercano a los $20 \mathrm{Km}$.

En este recorrido, habremos ido encontrando afloramientos de los materiales mesozoicos del Sistema Ibérico, que constituyen aquí, la Sierra de Gúdar. Así, habremos visto afloramientos de los materiales carbonatados del Cretácico, fundamentalmente.

Sin embargo, al llegar a las cercanías de Rubielos de Mora, habremos entrado en la Depresión de la Puebla de Valverde-Sarrión en su Cubeta de mora y habremos empezado a encontrar afloramientos arcillosos (a veces algo arenosos) y calcolutíticos del Mioceno. Estos son los materiales que aparecen en el lugar de la presente parada.

Precisamente, sobre estos materiales se han situado unos interesantes depósitos petrolíferos (de niveles bituminosos, concretamente). Éstos han estado en explotación a inicios y mediados del pasado siglo XX. Sin embargo, esta explotación no tuvo mucho éxito. Se inició la misma en el año 1918, a cargo de la empresa catalana Sociedad Sabadell y Henry. (fotografías 15 y 16).

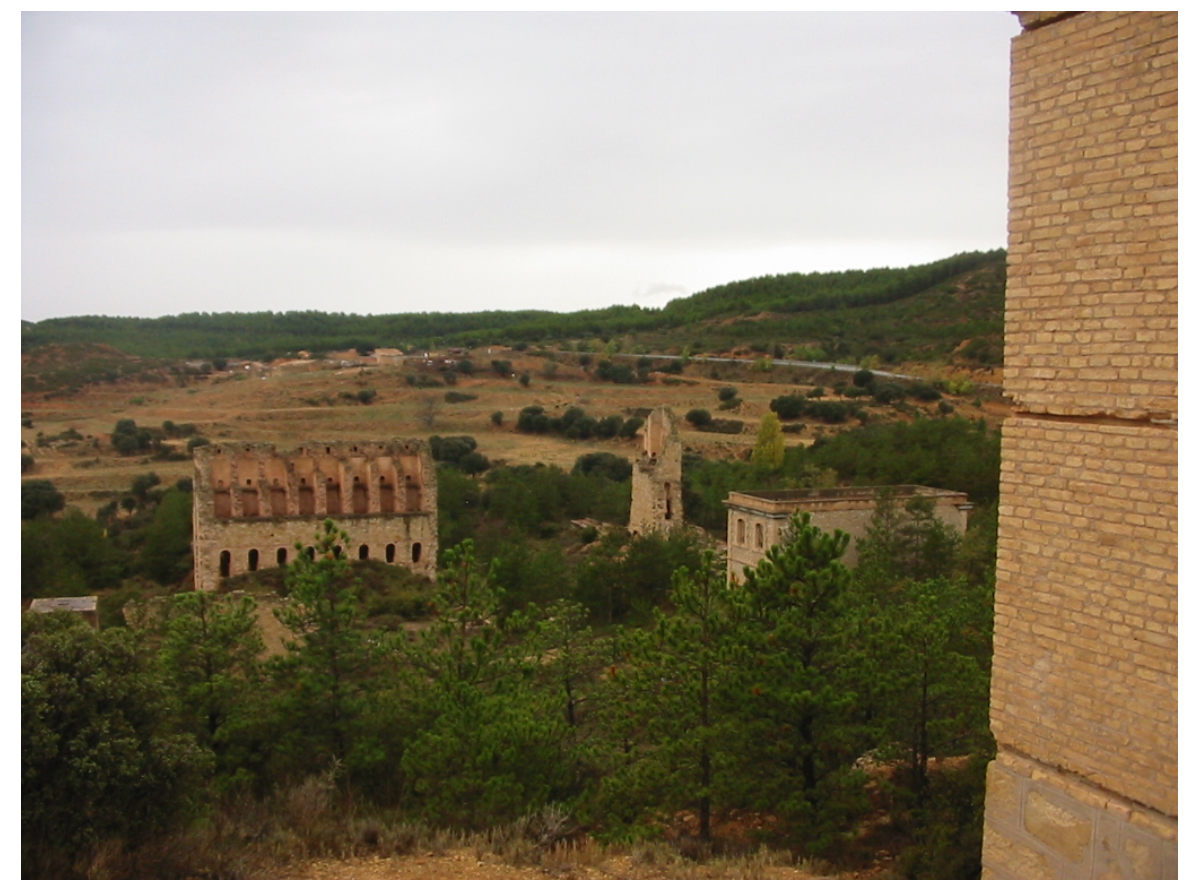

Fotografía 15. Un aspecto general de la zona minera bituminosa de Rubielos de Mora 


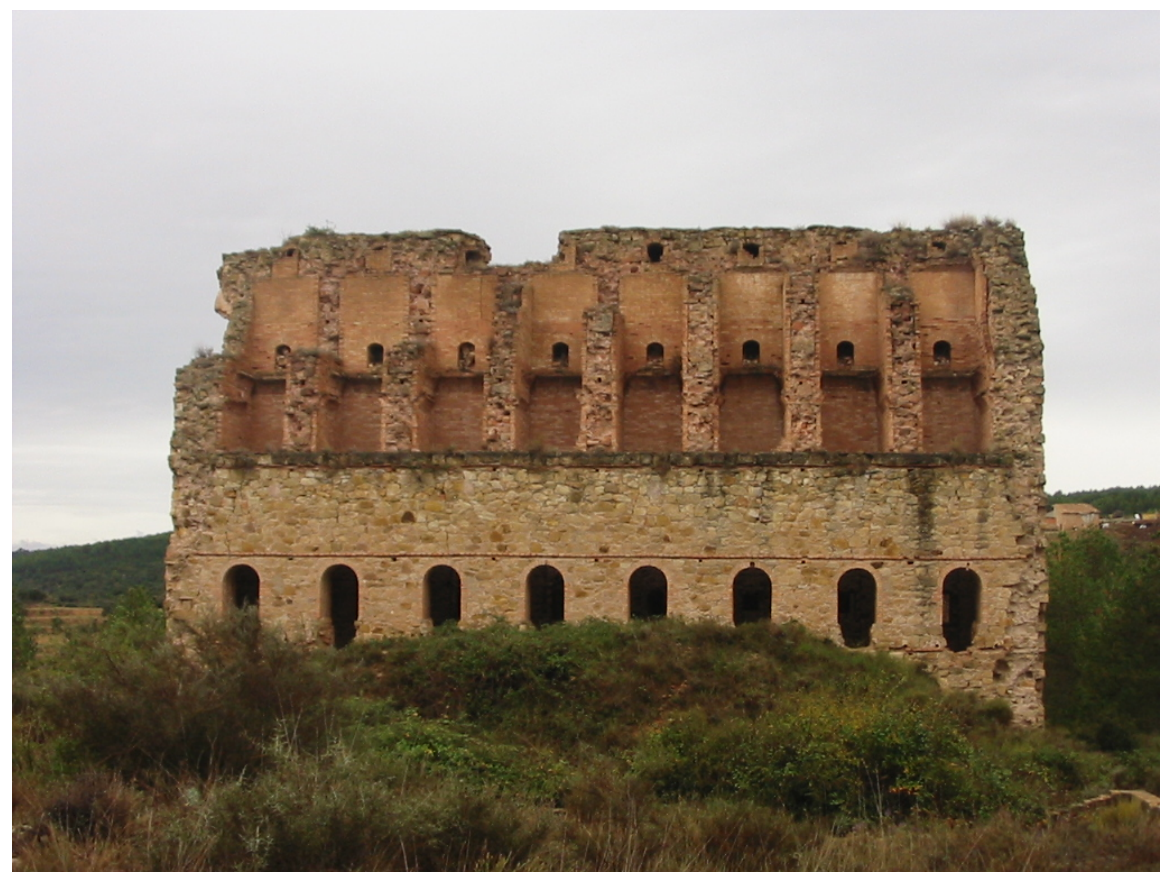

Fotografía 16. Zona de los hornos de calcinación de los materiales bituminosos Minas de Rubielos de Mora

\section{EN ESTE LUGAR FINALIZA EL RECORRIDO DEL PRESENTE ITINERARIO}




\section{BIBLIOGRAFIA}

CALVO, M. Et altri (1988).- Minerales de Aragón. Colección: temas geológicos, Edit. Mira, 210 pag. Zaragoza.

GADMA. (2001).- Puntos de Interés Geológico de Aragón, Edit. Gobierno de Aragón, Departamento de Medio Ambiente (GADMA). 243 pag. Zaragoza.

MAESTRE, A. (1845).- Descripción geognóstica del Distrito Minero de Aragón y Cataluña. Anales de Minas, t. III, Madrid.

MATA-PERELLÓ, J.M. (1991).- Inventario Mineralógico de la comarca de Gúdar - Javalambre. Rodeno, $n^{\circ} 34.26$ pag. Manresa.

MATA-PERELLó, J.M. (1991).- Inventario Mineralógico de la Región del Túria (Sectores Orientales). Rodeno, $\mathrm{n}^{\circ}$ 36. 42 pag. Manresa.

MATA-PERELLÓ, J.M. (2003).- Recorrido geológico y mineralógico por las comarcas de la Comunidad de Teruel, de Gúdar-Javalambre y de la Tierra de Montalbán: desde Teruel y la Puebla de Valverde a Linares de Mora y a Utrillas. Inédito, 12 pag. Manresa.

MATA-PERELLÓ, J.M. (2014).- Recorregut de recerca geològica i mineralògica per la comarca valenciana de l'Alt Millars i per l'aragonesa de Gúdar - Javalambre: des de Montanejos a Olba y a Rubielos de Mora. Inédito. 10 páginas. Manresa.

PEÑA MONGE, J.L. et altri (1984).- Geomorfología de la provincia de Teruel. Pub. Inst. Estudios Turolenses, 149 pag. Teruel. 